\title{
Artropodofauna associada à cagaiteira (Eugenia dysenterica DC., Myrtaceae) em coleção de germoplasma ex situ em Goiás, Brasil
}

\author{
Arthropodfauna associated with cagaiteira (Eugenia dysenterica DC., Myrtaceae) \\ in the collection of ex situ germoplasm in Goiás state, Brazil
}

\section{Charlles Brandão Silva Rocha', Jaqueline Magalhães Pereira' ${ }^{\mathrm{II}}$, Jéssica Ferreira Silva ${ }^{\mathrm{III}}$, André Júnio Andrade Peres ${ }^{\mathrm{III}}$, Rodrigo Damasco Daud ${ }^{\text {IV }}$}

\begin{abstract}
Resumo
Eugenia dysenterica é uma espécie nativa do Cerrado com potencial para exploração econômica em função do seu uso na alimentação. No entanto, estudos são necessários para possibilitar a domesticação da espécie em plantio comercial. Atualmente, os frutos são obtidos a partir do extrativismo em áreas do Bioma Cerrado. A artropodofauna associada à Eugenia dysenterica é praticamente desconhecida. Assim, o presente estudo teve como objetivo avaliar a artropodofauna associada às folhas de Eugenia dysenterica. Avaliamos quinzenalmente a artropodofauna em plantas de Eugenia dysenterica na coleção de germoplasma ex situ da Escola de Agronomia, UFG. Para tanto, três ramos do terço médio foram amostrados em 16 plantas. Posteriormente, sete folhas/ramo foram selecionadas e analisadas sob microscópio estereoscópio. As cochonilhas foram os artrópodes mais abundantes em Eugenia dysenterica. A presença de folhas novas no mês de outubro coincidiu com a eclosão das ninfas da cochonilha Ceroplastes acutus e com o aparecimento de galhas foliares causadas por dípteros. A superfamília Coccoidea e a família Aleyrodidae foram classificadas como superabundantes e superfrequentes. Duas famílias de Thysanoptera foram encontradas: Thripidae e Phlaeothripidae. Com relação aos ácaros, as espécies fitófagas foram as mais abundantes, seguidas pelos ácaros predadores. As famílias Phytoseiidae e Tenuipalpidae foram as mais representativas nas amostras. Aleirodídeos, dípteros e a cochonilha Ceroplastes acutus estão associados à emissão de novas folhas da cagaiteira. Este é o primeiro relato de ocorrência de espécies de ácaros, tripes e da cochonilha Ceroplastes acutus em Eugenia dysenterica.
\end{abstract}

Palavras-chave: Ácaros; Aleyrodidae; Cochonilha; Tripes

Engenheiro Florestal, Universidade Federal de Goiás, Av. Esperança, s/n, Campus Samambaia, CEP 74690-900, Goiânia (GO), Brasil. charlles. brandaosr@gmail.com (ORCID: 0000-0001-9635998X)

II Engenheira Agrônoma, Drª ., Professora do Setor Fitossanitário, Escola de Agronomia, Universidade Federal de Goiás, Av. Esperança, s/n, Campus Samambaia, CEP 74690-900, Goiânia (GO), Brasil. jmpereira@ufg.br (ORCID: 0000-0002-4911-555X)

III Engenheiro(a) Agrônomo(a), Doutorando(a), Programa de Pós Graduação em Agronomia, Universidade Federal de Goiás, Av. Esperança, s/n, Campus Samambaia, CEP 74690-900, Goiânia (GO), Brasil. jessicaferreira.agronoma@gmail.com (ORCID: 0000-0001-8984-5621)/ andrejaperes@ yahoo.com (ORCID: 0000-0002-7964-0611)

Iv Biólogo, Dr., Professor do Departamento de Ecologia, Instituto de Ciências Biológicas, Universidade Federal de Goiás, Av. Esperança, s/n, Campus Samambaia, CEP 74001-970, Goiânia (GO), Brasil. rodrigodaud36@gmail.com (ORCID: 0000-0002-5780-972X) 


\begin{abstract}
Eugenia dysenterica is a native species of the Cerrado biome which has potential for economic exploitation due to its economic importance in food. However, further studies are crucial to enable the domestication of this species in commercial plantation. Actually, the fruits are obtained by extractivism in area of the Cerrado vegetation. The arthropodfauna associated to Eugenia dysenterica is practically unknown. Thus, the present study aimed to evaluate the arthropodofauna associated with leaves of Eugenia dysenterica. We evaluated the arthropodfauna biweekly in Eugenia dysenterica plants in the collection of ex situ germoplasm of 'Escola de Agronomia', UFG. Three branches of the middle third were sampled in 16 plants. Seven leaves/branch were selected and analyzed under stereomicroscope. Cochineals were the most abundant arthropods in Eugenia dysenterica. The presence of new leaves on the plants, October, coincided with the hatching of the nymphs of the cochineal Ceroplastes acutus and the appearance of leaf galls caused by Diptera. The superfamily Coccoidea and the family Aleyrodidae were classified as superabundant and superfrequent. Two families of Thysanoptera were reported: Thripidae and Phlaeothripidae. In relation to plant mites, phytophagous species were the most abundant ones, followed by predatory mites. Families Phytoseiidae and Tenuipalpidae were the most representative in the samples. Aleyrodidae, Diptera and cochineal Ceroplastes acutus are associated with the emission of new leaves of Cagateira. This is the first report of the occurrence of mite species, thrips and Ceroplastes acutus in Eugenia dysenterica.
\end{abstract}

Keywords: Mites; Aleyrodidae; Cochineal; Thrips

\title{
Introdução
}

A cagaiteira (Eugenia dysenterica DC.) é uma planta nativa do Cerrado que possui casca suberosa e fendida, com aparência de blocos bem definidos e sobrepostos (SCARIOT; RIBEIRO, 2015). O fruto apresenta aspecto globoso, coloração amarela e a polpa é levemente ácida (ALVES, 2013) e devem ser consumidos com moderação, uma vez que podem causar diarreia e embriaguez (MATOS; QUEIROZ, 2009). Apresenta importância econômica principalmente pelo potencial alimentício dos seus frutos (CAMILO et al., 2014).

Dentre os insetos associados à cagaiteira podem ser destacadas as moscas frugívoras, que ocasionam danos em média de $73,94 \%$ dos frutos, caracterizados pelo aspecto de cicatrizes pontuais, pouco deprimidas na superfície externa, uma progressiva perda de consistência e ruptura das fibras internas, resultando no amolecimento dos frutos (FERREIRA et al., 2016). Quatro espécies do gênero Neosilba (Diptera: Lonchaeidae) foram relatadas em frutos de cagaiteira (GISLOTI; UCHOA; PRADO, 2017). Em ramos e folhas de espécies do gênero Eugenia também já foram previamente relatadas a formação de galhas (CARVALHO-FERNANDES et al., 2016), besouros desfolhadores (ARNHOLD; GONÇALVES, 2010) e lagartas desfolhadoras, tais como Eupseudosoma aberrans Schaus, Eupseudosoma involuta Sepp (Lepidoptera: Arctiidae), Euselasia apisaon Dalman (Lepidoptera: Riodinidae) e Thyrinteina amobia Stoll (Lepidoptera: Geometridae) (ZANUNCIO, 1993).

A cagaiteira é uma das espécies do Cerrado com grande potencial para a domesticação. Porém, existe uma escassez de informações básicas que dificulta o cultivo. Ainda é necessário avaliar, por exemplo, quais espécies de artrópodes são associadas à cagaiteira e quais delas poderiam apresentar surtos populacionais ou apresentarem danos à espécie vegetal. Por outro lado, também é relevante reconhecer os potenciais inimigos naturais a fim de possibilitar futuros programas de manejo em cultivos de cagaiteira. Em razão disso, no presente estudo foi avaliada a artropodofauna associada à cagaiteira, com ênfase nos insetos e ácaros presentes nas folhas dessa espécie vegetal. 


\section{Material e método}

A pesquisa foi conduzida na coleção de germoplasma ex situ de cagaiteiras da Escola de Agronomia, da Universidade Federal de Goiás (EA/UFG), em Goiânia, GO, (16³5'12"S, 49²1'14”'O, $730 \mathrm{~m}$ de altitude). A área de estudo é composta por coleções originárias das regiões Sudeste e Norte/Nordeste de Goiás. Os dados climáticos (Figura 1) foram obtidos da estação evaporimétrica da Escola de Agronomia - UFG, Goiânia, Goiás (1641”S e 49¹7”O, $741 \mathrm{~m}$ de altitude), localizada a 1.250 metros de distância da área de estudo.

\section{Figura 1 - Dados climáticos mensurados de janeiro a dezembro de 2016 a partir da estação evaporimétrica da Escola de Agronomia, UFG, Goiânia, GO}

Figure 1 - Climatological data on January to December 2016 evaporimeter station of the Escola de Agronomia, UFG, Goiânia, GO state

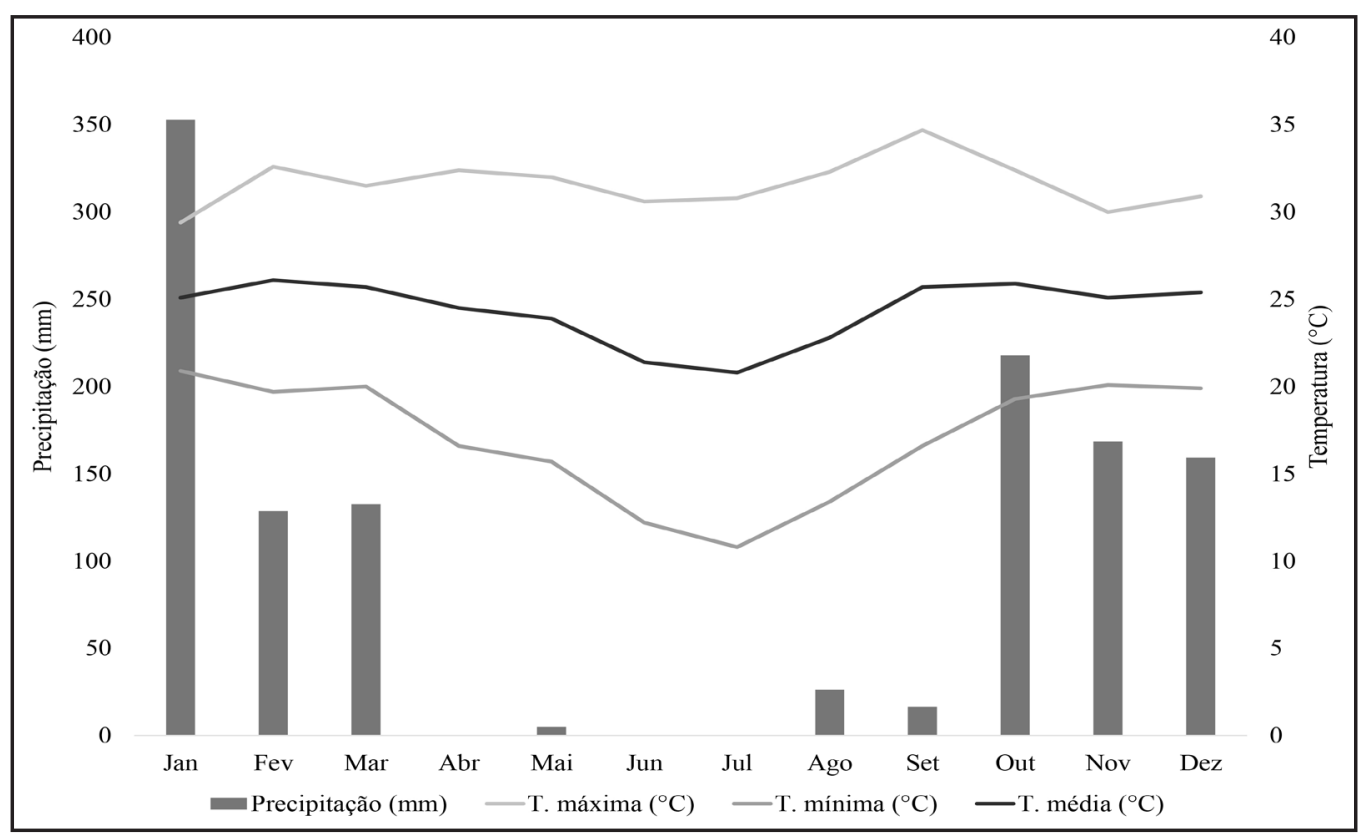

Fonte: www.agro.ufg.br.

Foram realizadas amostragens quinzenais durante o período de janeiro a dezembro de 2016. Dessa forma, foram coletados aleatoriamente três ramos do terço médio de 16 plantas para avaliação da artropodofauna associada à cagaiteira. As amostras foram acondicionadas em sacos plásticos, posteriormente encaminhadas ao laboratório e mantidas sob refrigeração a $10^{\circ} \mathrm{C}$ por um período máximo de uma semana. Foram selecionadas e analisadas sete folhas e seus ramos de cada planta com o auxílio de microscópio estereoscópio.

Os ácaros e tripes foram capturados com o auxílio de um pincel e transferidos para tubos tipo eppendorf, com álcool 70\%. Após a triagem, os ácaros foram montados em lâminas de microscopia como meio de Hoyer (MORAES; FLECHTMANN, 2008). A identificação e a contagem dos ácaros foram realizadas sob microscópio de contraste de fases com o auxílio de chaves de identificação (MORAES; FLECHTMANN, 2008) e consulta a especialistas. Os tripes foram montados em lâminas permanentes por meio de bálsamo do Canadá (MONTEIRO; MOUND, 2012). A identificação de tripes foi realizada por meio de chaves taxonômicas de Palmer, Mound e Du Heaume (1989) e através de comparações com exemplares identificados.

Após a identificação, os ácaros encontrados foram organizados em grupos de acordo 
com o hábito alimentar em predadores, fitófagos ou micófagos, seguindo informações sobre hábito alimentares disponíveis em literatura especializada (MORAES; FLECHTMANN, 2008; KRANTZ; WALTER, 2009). Para os demais artrópodes, a identificação e contagem foram realizadas diretamente sob estereomicroscópio.

Para análise faunística foram determinados os índices de abundância e frequência através do software ANAFAU. Os critérios aplicados pelo software para classificar os índices faunísticos foram:

Abundância (A) - Refere-se ao número de indivíduos por unidade de superfície ou volume e varia no espaço e no tempo. Foi classificada nas seguintes categorias: muito abundante (ma) número de indivíduos maior que o limite superior do intervalo de confiança da média; abundante (a) - número de indivíduos entre os limites superiores do intervalo de confiança; comum (c) número de indivíduos entre os limites inferior e superior do intervalo de confiança da média; dispersa (d) - número de indivíduos entre os limites inferiores do intervalo de confiança da média e rara $(r)$ - número de indivíduos menor que o limite inferior ao intervalo de confiança da média.

Frequência (F) - Refere-se ao cálculo da porcentagem dos indivíduos de uma dada espécie em relação ao total de indivíduos coletados. Foi classificada nas seguintes categorias: muito frequente $(\mathrm{mf})$ - frequência maior que o limite superior do intervalo de confiança da média; frequente (f) - frequência entre os limites inferior e superior do intervalo de confiança da média e pouco frequente (pf) - frequência menor que o limite inferior do intervalo de confiança da média.

\section{Resultados e discussão}

Foram amostrados 5.460 artrópodes pertencentes a classe Insecta (Hemiptera, Psocoptera, Diptera, Thysanoptera e Neuroptera) e a classe Arachnida (Araneae, Parasitiformes e Acariformes). A ordem Hemiptera apresentou o maior número de indivíduos $(87,30 \%)$, seguido pelos ácaros (Parasitiformes e Acariformes, 3,78\%), Psocoptera (3,67\%), Diptera (2,77\%), Thysanoptera (1,06\%), Araneae $(0,81 \%)$ e Neuroptera $(0,61 \%)$ (Tabela 1$)$. No total, 4.097 artrópodes foram identificados em nível de espécie e 83 em nível de gênero.

Tabela 1 - Sazonalidade de artrópodes encontrados em Eugenia dysenterica. Goiânia, GO, 2016

Table 1 - Seasonal arthropods reported in Eugenia dysenterica. Goiânia, GO state, 2016

\begin{tabular}{|c|c|c|c|c|c|c|c|c|c|c|c|c|c|c|}
\hline \multirow{2}{*}{$\begin{array}{c}\text { Superordem/ } \\
\text { Ordem }\end{array}$} & \multirow{2}{*}{$\begin{array}{c}\text { Família/ } \\
\text { Superfamília }\end{array}$} & \multicolumn{12}{|c|}{ Meses } & \multirow{2}{*}{ Total } \\
\hline & & $\mathbf{J}$ & $\mathbf{F}$ & $\mathbf{M}$ & A & $\mathbf{M}$ & $\mathbf{J}$ & $\mathbf{J}$ & $\mathbf{A}$ & $S$ & $\mathbf{O}$ & $\mathbf{N}$ & D & \\
\hline \multirow[t]{6}{*}{ Acariformes } & Iolinidae & - & 1 & - & - & - & - & - & - & - & - & - & - & 1 \\
\hline & Stigmaeidae & - & 1 & - & - & - & - & 1 & - & - & - & - & - & 2 \\
\hline & Tenuipalpidae & 1 & 10 & - & - & - & 6 & 11 & 13 & - & - & 20 & 10 & 81 \\
\hline & Tetranychidae & - & - & - & 1 & 1 & 3 & 2 & - & - & 1 & - & 1 & 9 \\
\hline & Tydeidae & - & - & 1 & - & - & - & - & 1 & - & - & 2 & - & 4 \\
\hline & Winterschmidtiidae & - & 1 & 2 & - & 1 & 6 & 23 & 1 & - & - & - & 1 & 35 \\
\hline \multirow[t]{2}{*}{ Parasitiformes } & Ascidae & 1 & 1 & - & - & - & - & - & - & - & - & - & - & 2 \\
\hline & Phytoseiidae & 3 & 7 & 15 & 15 & 14 & 9 & 10 & 8 & - & - & 1 & - & 82 \\
\hline Araneae & - & 1 & 22 & 3 & 1 & 3 & 3 & 6 & - & - & 3 & 1 & 1 & 44 \\
\hline Diptera & - & 11 & 2 & 10 & 10 & 7 & 2 & 5 & 1 & 27 & 48 & 6 & 22 & 151 \\
\hline
\end{tabular}


Tabela 1 - Conclusão ...

Table 1 - Conclusion ...

\begin{tabular}{lcccccccccccccc}
\hline $\begin{array}{c}\text { Superordem } \\
\text { Ordem }\end{array}$ & $\begin{array}{c}\text { Família/ } \\
\text { Superfamília }\end{array}$ & $\mathbf{~ J}$ & $\mathbf{F}$ & $\mathbf{M}$ & $\mathbf{A}$ & $\mathbf{M}$ & $\mathbf{J}$ & $\mathbf{J}$ & $\mathbf{A}$ & $\mathbf{S}$ & $\mathbf{O}$ & $\mathbf{N}$ & $\mathbf{D}$ & Total \\
\cline { 3 - 13 } Hemiptera & Aleyrodidae & 28 & 65 & 65 & 79 & 80 & 59 & 35 & 40 & 40 & 136 & 132 & 84 & 833 \\
& Coccoidea & 7 & 403 & 4 & 3 & 17 & 14 & 124 & 20 & 3 & 3314 & 11 & 5 & 3925 \\
Neuroptera & Chrysopidae & 2 & 3 & - & 1 & 2 & 2 & 1 & - & 1 & 4 & 11 & 6 & 33 \\
Psocoptera & - & - & 9 & 12 & 34 & 42 & 55 & 15 & 17 & - & - & 1 & 15 & 200 \\
Thysanoptera & Phlaeothripidae & - & 2 & - & - & 17 & 11 & 11 & - & 2 & 2 & 6 & 6 & 57 \\
& Thripidae & - & - & - & - & 1 & - & - & - & - & - & - & - & 1 \\
\hline Total & & 54 & 527 & 102 & 144 & 185 & 170 & 244 & 101 & 73 & 3508 & 191 & 151 & 5460 \\
\hline
\end{tabular}

Fonte: Autores (2019)

Apesar do número de indivíduos encontrados, apenas duas superordens e seis ordens foram registradas. Outros estudos com mirtáceas relatam maior diversidade de ordens encontradas. Para Eucalyptus sp. (Myrtaceae), Silva et al. (2012) registraram 936 indivíduos distribuídos em 12 ordens.

As cochonilhas foram os artrópodes mais abundantes nas amostras coletadas, tendo a espécie Ceroplastes acutus Peronti (Hemiptera: Coccidae) como destaque. Essa espécie foi previamente relatada em Machaerium acutifolium (Fabaceae) (PERONTI; SOUZA-SILVA, WILLINK, 2008) e em Psidium sp. (Myrtaceae) (CULIK et al., 2011).

O ciclo biológico de Ceroplastes acutus na cagaiteira foi semelhante ao encontrado por Hickel, Botton e Schuck (2010) para a espécie Parthenolecanium persicas Fabricius (Hemiptera: Coccidae) em cultivos de videira. Os autores registraram eclosão de ninfas no período de outubro a dezembro, quando se deslocam para as folhas em direção a nervura principal. Essas ninfas durante os meses de janeiro a fevereiro sofrem mais uma muda (segundo instar), continuando localizadas nas nervuras das folhas até o período próximo da senescência das folhas. O inseto passa o inverno praticamente imóvel, no segundo instar e em setembro, localizadas nos ramos, passam para o terceiro instar e se tornam sésseis. Durante o mês de outubro as ninfas de terceiro instar transformam-se em fêmeas adultas, reiniciando o ciclo biológico. Após o término da oviposição, a fêmea morre, porém sua carapaça continua fixa e protegendo os ovos durante o período de incubação.

Os ácaros também foram representativos nas amostras em cagaiteira. Foram identificados 216 ácaros de 14 espécies de 8 famílias (Tabela 2). As famílias Phytoseiidae e Tenuipalpidae destacaram-se quanto ao número de indivíduos. Os ácaros fitófagos foram os mais abundantes, representando $41,67 \%$ do material, seguido pelos predadores (40,28\%), micófagos (16,20\%) e hábito alimentar desconhecido (1,85\%). O predomínio de ácaros fitófagos sobre predadores sugere que a cagaiteira serve como refúgio e alimento para esse grupo de ácaros.

Rezende e Lofego (2011), através de estudos em plantas do bioma Cerrado, observaram que os fitoseídeos são os ácaros predadores predominantes na família Myrtaceae. Euseius concordis foi o predador mais representativo da família Phytoseiidae, representando $75,60 \%$ do total encontrado na cagaiteira. O baixo número de ácaros dessa espécie no mês de setembro e nos três meses consecutivos pode estar relacionado ao enfolhamento e florescimento das plantas. Com a queda das folhas, os indivíduos de Euseius concordis podem ter migrado em direção às flores para se alimentar de pólen ou para outras plantas hospedeiras em busca de presas. Ácaros do gênero Euseius são generalistas que preferem consumir pólen, mas podem se alimentar de ácaros e insetos (MCMURTRY; MORAES; SOURASSOU, 2013). 
Tabela 2 - Ácaros encontrados em folhas de Eugenia dysenterica. Goiânia, GO, 2016

Table 2 - Mites reported on leaves of Eugenia dysenterica. Goiânia, GO state, 2016

\begin{tabular}{lccc}
\multicolumn{1}{c}{ Família } & Gênero/Espécie & Hábito alimentar & No de indivíduos \\
\hline Ascidae & Asca sp. & Predador & 2 \\
Iolinidae & sp. & Predador & 1 \\
Phytoseiidae & Amblyseius hexadens & Predador & 11 \\
& Euseius concordis & Predador & 62 \\
Stigmaeidae & Iphiseiodes zuluagai & Predador & 9 \\
Tenuipalpidae & Agistemus sp. & Predador & 2 \\
& Brevipalpus sp. & Fitófago & 2 \\
Tetranychidae & Brevipalpus yothersi & Fitófago & 79 \\
& Eutetranychus banksi & Fitófago & 3 \\
& Oligonychus sp. & Fitófago & 3 \\
Tydeidae & Tetranychus mexicanus & Fitófago & 3 \\
Winterschmidtiidae & Afrotydeus smileyi & NCenspinskia sp. & Micófago \\
\hline
\end{tabular}

Fonte: Autores (2019)

Em que: NC = Hábito alimentar não conhecido

O maior número de aleirodídeos foi encontrado em outubro, após o enfolhamento da cagaiteira (Tabela 1). Dentre os insetos da ordem Thysanoptera foram identificados um exemplar de Heliothrips haemorrhoidalis Bouché (Thripidae) e uma nova espécie do gênero Pseudophilothrips (Phlaeothripidae), que está em fase de descrição. O gênero Pseudophilothrips foi o táxon de Thysanoptera com o maior número de indivíduos amostrados. Em eucalipto, mesma família da cagaiteira, já foram previamente registradas as espécies de tripes Selenothrips rubrocinctus Giard, Retithrips syriacus Mayet e Thrips australis Bagnall (MONTEIRO; MOUND; ZUCCHI, 1999; 2001). Affeld et al. (2010) relataram a ocorrência de três famílias de tripes (Merothripidae, Phlaeothripidae e Thripidae) em Metrosideros robusta (Myrtaceae). Pinent et al. (2005) avaliaram espécies de tripes associadas à família Myrtaceae e registraram Heliothrips haemorrhoidalis, em plantas da espécie Eugenia hiemalis. Esse fato sugere que essa espécie de tripes utiliza outras espécies do gênero Eugenia como hospedeira.

A partir das análises faunísticas, a superfamília Coccoidea e a família Aleyrodidae foram consideradas as mais representativas, ocorrendo como superabundantes e superfrequentes. As ordens Psocoptera e Diptera também foram expressivas, ocorrendo como muito abundantes e muito frequentes. Em contrapartida, a menor representatividade foi demonstrada pelas famílias Thripidae, Ascidae, Iolinidae, Stigmaeidae e Tydeidae, ocorrendo como raras e pouco frequentes (Tabela 3). 
Tabela 3 - Índices faunísticos das superordens e ordens, famílias e superfamília da artropodofauna encontrada na Eugenia dysenterica. Goiânia, GO, de janeiro a dezembro de 2016

Table 3 - Faunistic indexes of the superorders and orders, families and superfamily of the arthropodofauna reported in Eugenia dysenterica, Goiânia. GO state, January to December 2016

\begin{tabular}{lcccc}
\hline Superordem/Ordem & Família/Superfamília & Número de indivíduos & A & F \\
\hline Acariformes & Iolinidae & 1 & r & pf \\
& Stigmaeidae & 2 & r & pf \\
& Tenuipalpidae & 71 & c & f \\
& Tetranychidae & 9 & d & pf \\
& Tydeidae & 4 & r & pf \\
& Winterschmidtiidae & 35 & c & f \\
Parasitiformes & Ascidae & 2 & r & pf \\
& Phytoseiidae & 82 & a & mf \\
Araneae & - & 44 & c & f \\
Diptera & - & 151 & ma & mf \\
Hemiptera & Aleyrodidae & 883 & as & sf \\
& Coccoidea & 3925 & sa & sf \\
Neuroptera & Chrysopidae & 33 & c & f \\
Psocoptera & - & 200 & ma & mf \\
Thysanoptera & Phlaeothripidae & 57 & c & f \\
\hline & Thripidae & 1 & r & pf \\
\hline
\end{tabular}

Fonte: Autores (2019)

Em que: A= Abundância - (sa) superabundante; (ma) muito abundante; (a) abundante; (c) comum; (d) dispersa; $(\mathrm{r})$ rara. F= Frequência - (sf) superfrequente; $(\mathrm{mf})$ muito frequente; (f) frequente; (pf) pouco frequente.

O enfolhamento da cagaiteira ocorreu durante todo o ano, com renovação total das folhas no início de outubro, coincidindo com a eclosão das ninfas de Ceroplastes acutus e com o surgimento de galhas foliares (Diptera) e de aleirodídeos. A maior parte das galhas foliares encontradas apresentou coloração verde, forma elipsoide, sem pilosidade e uma câmara interna. Foram identificadas como dípteros devido à observação de características da fase larval, porém não foram obtidos adultos. Carvalho-Fernandes et al. (2016) realizaram estudos sobre insetos galhadores e encontraram maior abundância em plantas do gênero Eugenia, correspondendo a $60 \%$ das galhas encontradas na família Myrtaceae, com 21 morfotipos de galhas. Lindner, Mendonça Junior e Cavalleri (2016) relatam galhas foliares ocasionadas por Holopothrips molzi Lindner (Thysanoptera: Phlaeothripidae) em Myrcia guianensis (Myrtaceae).

O início da emissão de folhas novas foi posterior à queda total das folhas velhas no final de setembro. Todas as plantas avaliadas apresentaram-se totalmente desfolhadas no período. A floração da cagaiteira ocorreu no mês de setembro e a frutificação ocorreu em outubro, aproximadamente 30 dias após a floração. Em outubro foi verificado pico populacional de artrópodes em cagaiteira (Figura 2). Nos meses de janeiro e setembro foram encontradas as menores quantidades de artrópodes (Figura 2, Tabela 1). 


\section{Figura 2 - Flutuação populacional dos artrópodes amostrados de janeiro a dezembro de 2016 em Eugenia dysenterica, Goiânia, GO}

Figure 2 -Population fluctuation of arthropods sampled to January to December 2016 in

Eugenia dysenterica, Goiânia, GO state

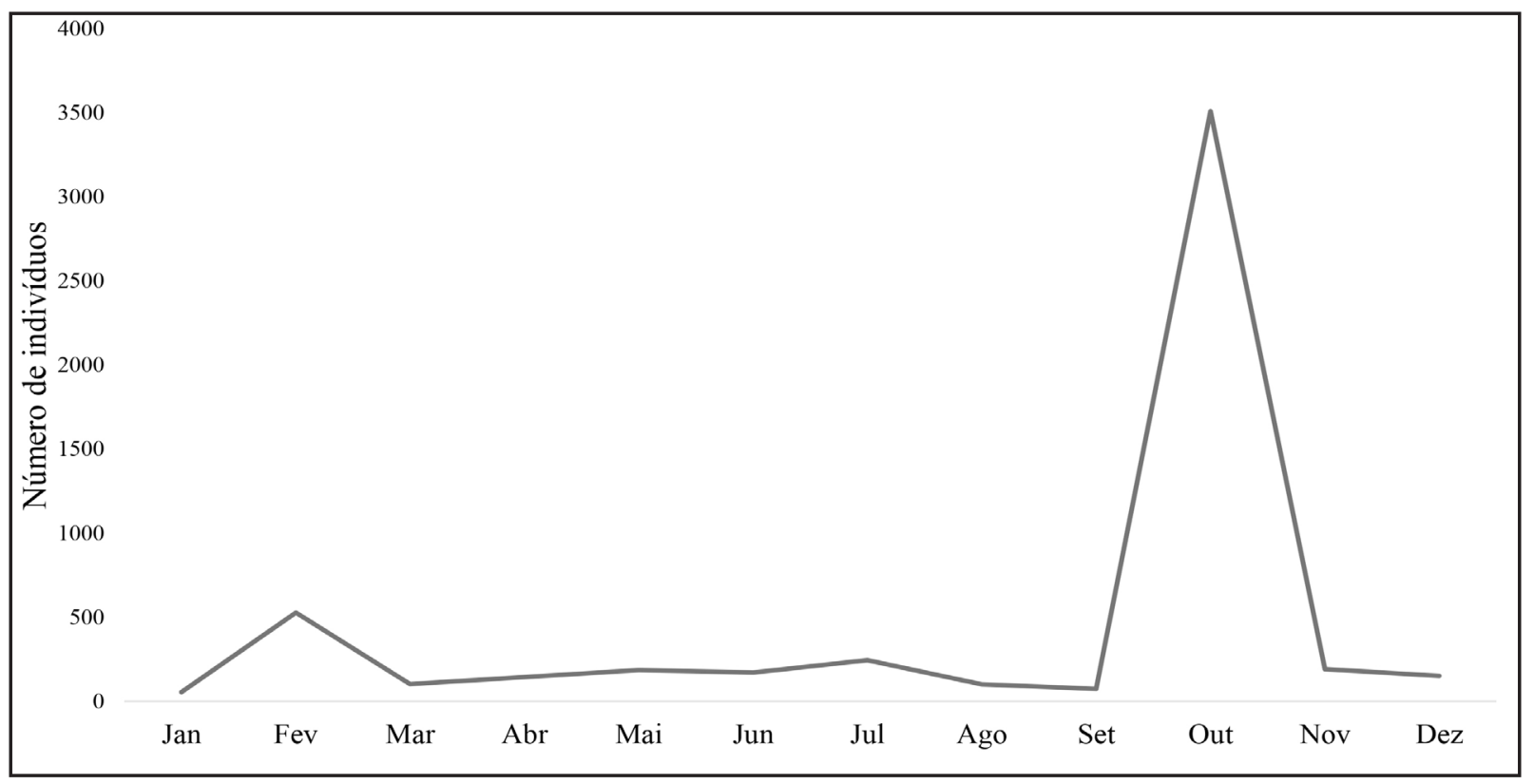

Fonte: Autores (2019)

A senescência natural da cagaiteira pode ter influenciado na baixa população de artrópodes no mês de setembro. A emissão de folhas novas ocorreu após a senescência, sendo que essas folhas apresentam coloração avermelhada. A cor vermelha em folhas jovens está associada com a presença de flavonoides (BOIÇA JÚNIOR et al., 2016). Além disso, compostos fenólicos, representados principalmente por taninos e flavonoides, detectados em folhas de Eugenia dysenterica (COUTO et al., 2009), podem apresentar funções múltiplas, incluindo a proteção das plantas contra a herbivoria de insetos (DE BORTOLI et al., 2012). Assim, a baixa abundância de artrópodes em setembro pode ser explicada pela ação desses compostos do metabolismo secundário durante o período.

Por outro lado, em relação ao mês de janeiro, o baixo número de artrópodes pode ter ocorrido por influência da precipitação. Atipicamente, no mês de janeiro choveu $353 \mathrm{~mm}$, mais do que o esperado $(244,5 \mathrm{~mm})$. Chuvas intensas aumentam a mortalidade dos insetos (PEREIRA et al., 2010). Além de atuar na mortalidade, a precipitação também pode afetar o potencial reprodutivo, influenciando a dinâmica populacional do inseto (AMORIM et al., 2011).

\section{Conclusões}

Aleirodídeos, dípteros e a cochonilha Ceroplastes acutus associaram-se à emissão de novas folhas de Eugenia dysenterica.

Este é o primeiro relato de ácaros, tripes e da cochonilha Ceroplastes acutus associados à Eugenia dysenterica. 


\section{Agradecimentos}

Ao Conselho Nacional de Desenvolvimento Científico e Tecnológico (CNPq) pela bolsa de iniciação científica concedida ao primeiro autor. A Fundação de Amparo à Pesquisa do Estado de Goiás (201200765800779, chamada pública n. 007/2012 - PRONEX - FAPEG) pelo auxílio financeiro concedido para o projeto de pesquisa. A equipe do Laboratório de Acarologia (UNESP - Campus São José do Rio Preto), pelo auxílio na identificação dos ácaros. Ao Prof. Dr. Élison Fabrício Bezerra Lima (UFPI - Campus Amilcar Ferreira Sobral) pelo auxílio na identificação dos indivíduos da ordem Thysanoptera. A Prof ${ }^{a}$. Drª . Ana Lúcia Benfatti Gonzalez Peronti (UNESP - Campus Jaboticabal) pela identificação da cochonilha Ceroplastes acutus.

\section{Referências}

AFFELD, K. et al. The invertebrate fauna of epiphyte mats in the canopy of northern rata (Myrtaceae: Metrosideros robusta A. Cunn.) on the West Coast of the South Island, New Zealand. Journal of Zoology, [s. l.], v. 36, n. 2, p. 177-202, 2010.

ALVES, M. A. Caracterização física e química, compostos bioativos e capacidade antioxidante de frutas nativas do Cerrado.2013. Dissertação (Mestrado em Ciências e Tecnologia de Alimentos) - Universidade Federal de Goiás, Goiânia, 2013.

AMORIM, H. C. S. et al. Mortalidade de Tuta absoluta por precipitação natural. In: CONGRESSO BRASILEIRO DE OLERICULTURA, 51., 2011, Viçosa, MG. Anais [...], [S. l.: s. n.], 2011. v. 2. p. 796-804.

ARNHOLD, A.; GONÇALVES, D. Ocorrência de Costalimaita lurida (Coleoptera: Chrysomelidae) em Eucalyptus spp. em Minas Gerais. Pesquisa Florestal Brasileira, [s. l.], v. 30, n. 63, p. 257-259, 2010.

BOIÇA JÚNIOR, A. L. et al. Resistência de plantas: explorando o potencial de defesa aos insetos. In: CASTILHO, R. C. et al. (ed.). Tópicos em Entomologia Agrícola - VIII. Jaboticabal: Gráfica Multipress, 2016. p. 73-94.

CAMILO, Y. M. V. et al. Caracterização de frutos e seleção de progênies de cagateiras (Eugenia dysenterica DC). Científica, Jaboticabal, v. 42, n. 1, p. 1-10, 2014.

CARVALHO-FERNANDES, S. P. et al. Diversity of insect galls associated with coastal shrub vegetation in Rio de Janeiro, Brazil. Anais da Academia Brasileira de Ciências, [s. l.], v. 88, n. 3, p. 1407-1418, 2016.

COUTO, R. O. et al. Caracterização físico-química do pó das folhas de Eugenia dysenterica DC. (Myrtaceae). Revista Eletrônica de Farmácia, [s. l.], v. 4, n. 3, p. 59-69, 2009.

CULIK, M. P. et al. Hemiptera, Coccoidea: Distribution extension and new records for the states of Espírito Santo, Ceará, and Pernambuco, Brazil. CheckList, [s. l.], v. 7, n. 4, p. 567-570, 2011.

DE BORTOLI, S. A. et al. Herbivoria em soja: efeito na composição química das folhas e na biologia da lagarta da soja e do percevejo verde pequeno. Comunicata Scientia, [s. l.], v. 3, n. 3, p. 192-198, 2012.

FERREIRA, G. A. et al. Caracterização de danos de moscas-das-frutas (Diptera: Tephritoidea) em frutos de Stenocalyx dysentericus (Mart.) O. Berg. Magistra, [s. l.], v. 28, n. 2, p. 261-267, 2016.

GISLOTI, L. J.; UCHOA, M. A.; PRADO, A. New records of fruit trees as host for Neosilba species (Diptera, Lonchaeidae) in southeast Brazil. Biota Neotropica, Campinas, v. 17, n. 1, 2017.

HICKEL, E. R.; BOTTON, M; SCHUCK, E. Pragas da videira e seu controle no estado de Santa Catarina. 2. ed. Florianópolis: EPAGRI, 2010.137 p. 
KRANTZ, G. W.; WALTER, D. E. A manual of acarology. 3th ed. Texas: Texas Tech University Press, 2009. 807 p.

LINDNER, M. F.; MENDONÇA JÚNIOR, M. S.; CAVALLERI, A. Holopothrips molzi sp. n. (Thysanoptera, Phlaeothripidae): Natural history and interactions in Myrtaceae galls. Zootaxa, [s. l.], v. 4114, n. 2, p. 139-148, 2016.

MATOS, E.; QUEIROZ, L. P. Árvores para cidades. Salvador: Ministério Público do Estado da Bahia, 2009. 340 p.

MCMURTRY, J. A.; MORAES, G. J. de; SOURASSOU, N. F. Revision of the lifestyles of phytoseiid mites (Acari: Phytoseiidae) and implications for biological control strategies. Systematic and Applied Acarology, [s. l.], v. 18, n. 4, p. 297-320, 2013.

MONTEIRO, R. C.; MOUND, L. A. Thysanoptera. In: RAFAEL, J. A. et al. Insetos do Brasil: diversidade e taxonomia. [S. l.]:Holos editora, 2012. p. 407-438.

MONTEIRO, R. C.; MOUND, L. A.; ZUCCHI, R. A. Espécies de tripes (Thysanoptera, Thripidae) no Brasil. Neotropical Entomology, [s. l.], v. 30, n. 1, p. 61-63, 2001.

MONTEIRO, R. C.; MOUND, L. A.; ZUCCHI, R. A. Thrips (Thysanoptera) as pests of plant production in Brazil. Revista Brasileira de Entomologia, [s. l.], v. 43, p. 163-171, 1999.

Moraes, G. J.; FleChtmann, C. H. W. Manual de Acarologia, Acarologia Básica e Ácaros de Plantas Cultivadas no Brasil. [S. l.]: Holoseditora, 2008. 308 p.

PALMER, J. M.; MOUND, L. A.; DU HEAUME, G. J. CIE guides to insects of importance to man: Thysanoptera. International Institute of Entomology, [s. l.], v. 1, n. 2, p. 55, 1989.

PEREIRA, A. I. A. et al. Fatores de mortalidade natural do Symphyta Neotropical Haplostegus nigricrus (Hymenoptera: Pergidae). Bioscience Journal, Uberlândia, v. 26, n. 1, p. 115-120, 2010.

PERONTI, A. L. B. G.; SOUSA-SILVA, C. R.; WILLINK, M. C. G. Revisão das espécies Cerosplastinae Atkinson (Hemiptera, Coccoidea, Coccidae) do Estado de São Paulo, Brasil. Revista Brasileira de Entomologia, [s. l.], v. 52, n. 2, p. 139-181, 2008.

PINENT, S. M. J. et al. Thysanoptera: plantas visitadas e hospedeiras no Parque Estadual de Itapuã, Viamão, RS, Brasil. Iheringia, Série Zoológica, [s. l.], v. 95, p. 9-16. 2005.

REZENDE, J. M.; LOFEGO, A. C. Phytoseiidae (Acari: Mesostigmata) on plants of the central region of the Brazilian Cerrado. Acarologia, [s. l.], v. 51, p. 449-463, 2011.

SCARIOT, A.; RIBEIRO, J. F. Boas práticas de manejo para o extrativismo sustentável da Cagaita. Brasília: EMBRAPA Recursos Genéticos e Biotecnologia, 2015. 72 p.

SILVA, R. I. da et al. Comparação da artropodofauna em monocultura de eucaliptos e Cerrado da FLONA no Distrito Federal. Ensaios e Ciência: Ciências Biológicas, Agrárias e da Saúde, [s. l.], v. 16, n. 2, p. 105-114, 2012.

ZANUNCIO, J. C. Manual de Pragas em Florestas - Lepidópteros desfolhadores de eucalipto: biologia, ecologia e controle. [S. l.]: IPEF; SIF, 1993. v. 1. 140 p. 